\title{
A dieta mediterrânica na prevenção de doenças da contemporaneidade: Uma revisão bibliográfica
}

\section{The Mediterranean diet in the prevention of contemporaneity diseases: A literature review}

\author{
Jorge Bonito
}

Universidade de Évora, Apartado 94, 7002-554 Évora, Portugal. Telef.: 266-768-050. Fax.: 266-768-073 E-mail: jbonito@uevora.pt

Resumo: O trabalho tem como objetivo apresentar evidências científicas existentes sobre a relação entre a dieta mediterrânica e a hipertensão, a hipercolesterolemia e as doenças cardiovasculares. Realizou-se através de uma revisão da literatura, usando-se a saturação com o critério para a exclusão de novos trabalhos. Os resultados apontam para o importante papel da dieta mediterrânica, ou outra de caraterísticas similares, junto com um estilo de vida fisicamente ativo que permita manter o peso dentro de um nível considerado saudável, na redução do risco de desenvolver doença cardiovascular, aumentando a qualidade e a esperança de vida. Um número superior a um terço das mortes causadas por doenças cardiovasculares são atribuídas aos hábitos alimentares e de atividade física e a outros fatores de risco estreitamente relacionados com os mesmos, como seja a hipertensão arterial, a aterosclerose, a diabetes e a obesidade. A maior parte das diferenças individuais do risco de se vir a desenvolver obesidade, cancro ou doenças cardiovasculares e das variações populacionais na incidência e na prevalência destas doenças deve-se a fatores relacionados com os estilos de vida.

Palavras-chaves: Alimentação saudável. Hipertenção arterial. Hipercolesterolemia. Doenças cardiovasculares.

\begin{abstract}
This work seeks to reveal the state of the art scientific evidence that exists on the relationship between the Mediterranean diet and hypertension, hypercholesterolemia and cardiovascular disease. It was held through a literature review, using saturation with the criteria for the exclusion of new articles. The results point to the important role of the Mediterranean diet, or one with similar characteristics, along with a physically active lifestyle for maintaining weight within a level considered healthy, in reducing the risk of developing cardiovascular disease, increasing quality and life expectancy. A number greater than one-third of deaths from cardiovascular diseases are attributed to the eating habits and physical activity and other risk factors closely related thereto, such as high blood pressure, atherosclerosis, diabetes and obesity. Most of the individual differences of the risk of eventually developing obesity, cancer or cardiovascular diseases and population variations in the incidence and prevalence of these diseases is due to factors related to lifestyles.
\end{abstract}

Keywords: Healthy eating. Arterial hypertension. Hypercholesterolaemia. Cardiovascular diseases. 


\section{INTRODUÇÃO}

Durante as últimas décadas, em Portugal, produziu-se um abandono progressivo da dieta mediterrânica, em paralelo a um aumento do consumo de alimentos processados altamente energéticos, que se caraterizam pelo seu alto conteúdo em gorduras de má qualidade, açúcares ou sal e pelo seu baixo conteúdo em outros nutrientes necessários para o desenvolvimento e a manutenção de um bom estado de saúde (DURÃO, OLIVEIRA; ALMEIRDA, 2008). Esta mudança de padrão alimentar está vinculada à ampla disponibilidade e à intensa pressão comercial para promover o consumo de alimentos processados altamente energéticos. Em 2010, a Organização Mundial de Saúde (OMS), consciente dos efeitos perniciosos da publicidade sobre os hábitos alimentares da população, e da infância em particular, incentivou os Estados Membros a promover políticas para reduzir o impacto sobre as crianças da promoção de alimentos ricos em gorduras saturadas, ácidos gordos insaturados hidrogenados (AGT), açúcares livres ou sal (WHO, 2010).

A pesquisa científica desenvolvida até ao momento já permitiu identificar 10 fatores major das doenças cardiovasculares (DCV). Nesta revisão damos particular atenção às relações entre a dieta, a hipertensão arterial (HTA) a dislipidémia e as DCV

\section{METODOLOGIA}

O método para a realização deste estudo foi a revisão bibliográfica, tendo-se utilizado na pesquisa as seguintes palavras chave: "ácidos gordos", "alimentação saudável", "cancro", "dieta mediterrânica", "dislipidémias", "hipertensão arterial" e "hipercolesterolemia". Usou-se como critério de exclusão, de novos trabalhos, a saturação da temática, quando se verificou não existirem aportes com novas evidências científicas. A pesquisa decorreu entre novembro de 2014 e fevereiro de 2015 e foi realizada utilizando como ferramenta a base de dados de revistas e outras publicações do Scielo e do Google académico, para além de sítios Web.

\section{REVISÃO BIBLIOGRÁFICA}

\section{Dieta e ácidos gordos hidrogenados}

As principais fontes de AGT são os óleos submetidos a processo de aquecimento ou a cozinha a altas temperaturas e, em menor dimensão, os produtos derivados da carne e do leite de vaca e de ovelha, por ação bacteriana no tubo digestivo dos ruminantes (biohidrogenação). Os AGT representam cerca de 5\% do total das gorduras contidas de forma natural nos produtos derivados de vaca e de ovelha, enquanto podem situar-se em volta de $50 \%$ as gorduras de produtos produzidos pela indústria mediante a hidrogenação de óleos vegetais. A importância dos AGT radica nos seus efeitos perniciosos para a saúde em geral, e a saúde cerebrocardiovascular em particular (MONTEIRO; ALMEIDA, 2007), uma vez que a ingestão de $5 \mathrm{~g}$ de AGT, quantidade que podemos encontrar com frequência numa porção de batatas fritas ou de um frango de um restaurante de comida rápida, associa-se a um incremento de $25 \%$ do risco de cardiopatia isquémica (STENDER; DYERBERG, 2006).

Para deterioração do padrão alimentar da população também contribui uma rotulagem alimentar deficiente. $\mathrm{O}$ etiquetado nutricional dos produtos processados altamente energéticos, ricos em gorduras de má qualidade, açúcares ou sal e pobres em nutrientes raramente apresenta a informação nutricional num formato facilmente legível e compreensível para muitos consumidores, omitindo o conteúdo dos nutrientes estreitamente relacionados com um maior risco de doenças crónicas, como sejam os AGT. O Traffic light labelling representa uma exceção notável, enquanto alternativa para as melhores escolhas alimentares (LIMA; ROSENTHAL; DELIZA， 2014; LONGO-SILVA; TOLONI; TADDEI, 2010).

Alguns governos também tomaram iniciativas neste domínio. O da Dinamarca, pioneiro, limitou o uso de AGT produzidos industrialmente a um máximo de $2 \%$ da gordura de qualquer produto alimentar. Foi seguido, em 2008, pelo Governo da Suíça. No Canadá, em junho de 2006, um comité do Ministério da Saúde recomendou um limite de 5\% de AGT (da gordura total) em todos os produtos vendidos aos consumidores (2\% para margarinas). Nos Estados Unidos da América, em 2003, a Food and Drug Administration emitiu um regulamento que obriga os fabricantes a listarem os AGT num painel de informações nutricionais nos alimentos e em alguns suplementos dietéticos. A nova regra de rotulagem tornouse obrigatória em toda a linha de produção. No Brasil, o Ministério da Saúde celebrou um acordo com representantes da indústria alimentar para reduzir a quantidade de AGT dos alimentos, resultando na remoção voluntária, em 2013, de cerca de 230 mil ton deste tipo de gordura dos alimentos processados. A recomendação da estratégia da OMS encontra-se descrita na $6 .^{\mathrm{a}}$ diretriz do "Guia Alimentar da População Brasileira". Não é sugerida a eliminação dos AGT, mas antes que exista uma restrição de um limite de consumo correspondente a $1 \%$ do valor energético diário total, o equivalente a cerca de $2 \mathrm{~g} / \mathrm{dia}$ numa dieta normal de $2 \mathrm{kcal}$ (BRASIL - MINISTÉRIO DA SAÚDE, 2008).

$\mathrm{Na}$ União Europeia (UE), foi pedido um parecer à Autoridade Europeia de Segurança Alimentar sobre os efeitos dos AGT na saúde e sobre as recomendações de consumo. O parecer de 2010 da EFSA (2010) considera que relativamente aos ácidos gordos saturados (AGS) e AGT a ingestão deve ser tão baixa quão possível, não se estabelecendo qualquer valor de referência nutricional para ácidos gordos monoinsaturados (AGM) e polinsaturados (AGP) cis.

Em síntese, ainda que os hábitos alimentares tenham a ver com decisões individuais, estas podem ser facilitadas ou dificultadas pelo meio sociopolítico, físico, económico e cultural onde cada um vive, sendo particularmente relevante no caso da infância, já que, perante conflitos de interesse, o direito das crianças em crescer num ambiente livre de influências negativas para a sua saúde deveria prevalecer sempre. A Convenção sobre os Direitos das Crianças (1989), ratificada por Portugal em 1990, estabelece que: 
“Os Estados Partes reconhecem à criança o direito de gozar do melhor estado de saúde possível e a beneficiar de serviços médicos e de reeducação. Os Estados Parte velam pela garantia de que nenhuma criança seja privada do direito de acesso a tais serviços de saúde (n. ${ }^{\circ} 1$ do art. $\left.^{\circ} 24 .^{\circ}\right)$. Os Estados Partes prosseguem a realização integral deste direito e, nomeadamente, tomam medidas adequadas para:assegurar que todos os grupos da população, nomeadamente os pais e as crianças, sejam informados, tenham acesso e sejam apoiados na utilização de conhecimentos básicos sobre a saúde e a nutrição da criança, as vantagens do aleitamento materno, a higiene e a salubridade do ambiente, bem como a prevenção de acidentes" (alínea e do $\operatorname{art}^{\circ}{ }^{24} 4^{\circ}$ ).

Para alcançarmos tal objetivo, além da responsabilidade individual, existe a responsabilidade coletiva em criar um meio que facilite as escolhas saudáveis, mediante medidas comunitárias (educativas, reguladoras, económicas) a nível internacional, nacional, local, laboral, escolar e familiar.

\section{Dieta e hipertensão arterial}

O sódio $(\mathrm{Na})$ é um nutriente essencial para o organismo humano. Contribui para a regulação osmótica dos fluidos e atua na condução de estímulos nervosos e na contração muscular. Tendo por base a massa molar do cloreto de sódio $(\mathrm{NaCl})$ e a massa molar do $\mathrm{NA}$, pode-se determinar a relação entre $\mathrm{NaCl}-\mathrm{Na}$, podendo-se, assim, concluir que $1 \mathrm{~g}$ deste sal contém $394 \mathrm{mg}$ de $\mathrm{Na}$, ou seja, aproximadamente $40 \%$.

A correlação positiva entre a ingestão de sal e a pressão arterial, reconhecida pela primeira vez há um século, tem sido bem estabelecida em estudos ecológicos, epidemiológicos, experimentais e humanos (WARDENER; MACGREGOR, 2002). Igualmente bem estabelecida é a associação do aumento da pressão arterial, da morbilidade e da mortalidade cardiovascular. A relação positiva da pressão arterial e as DCV e a associação positiva da ingestão de $\mathrm{Na}$ à pressão arterial, constituem a base da hipótese de que uma redução na ingestão de sal, em virtude do seu efeito hipotensor, pode prevenir derrames e ataques cardíacos. Além disso, mesmo se o efeito sobre a pressão sanguínea fosse da ordem da redução de 1-2 mm de $\mathrm{Hg}$, para cada 75-100 mmol de consumo de $\mathrm{Na}$ reduzido, o impacto de uma tal mudança, aplicada à totalidade população, seria enorme (ALDERMAN, 2000). O problema com esta possibilidade atraente é que a redução no consumo de sal desta magnitude tem outras e, por vezes, consequências adversas para a saúde. A questão, portanto, é se os efeitos hipotensores benéficos da restrição de $\mathrm{Na}$ superam seus riscos. Infelizmente, alguns dos dados disponíveis sobre os efeitos da baixa ingestão de $\mathrm{Na}$ nos resultados da saúde são inconsistentes.

Freedman e Petitti (2001) sustentam que a hipótese de que a ingestão de sal continuada incrementa a
HTA não é válida para a generalidade da população mundial. Contrapõem quatro estudos em populações do Quénia, Papua e de duas tribos indígenas do Brasil, onde se verifica baixa ingestão de sal e de pressão sanguínea com 48 estudos em populações onde as pressões sanguíneas são baixas e os consumos de sal são elevados. Consideram, assim, que a evidência científica sugere, apenas, uma modesta redução da pressão sanguínea com a diminuição do consumo de sal e que as posições assumidas pelas agências oficiais e revistas de medicina acerca da redução do consumo de sal espelham mais posições de políticas de saúde do que propriamente a evidência científica.

Em síntese, sem o conhecimento da soma dos múltiplos efeitos de uma dieta reduzida em $\mathrm{Na}$, nenhuma indicação universal sobre a ingestão de $\mathrm{Na}$ pode ser justificada cientificamente. Aceitemos, por ora, que o consumo excessivo de sal tem uma relação causal direta com a HTA, existindo evidência científica que constitui fator de risco para algumas populações. É, por conseguinte, um fator de risco para DCV em toda a Europa (TAYLOR; ASHTON; MOXHAM; HOOPER; EBRAHIM, 2011a), aumentando o risco de acidentes cerebrovasculares, de hipertrofia do ventrículo esquerdo e de doenças renais (HE; MARKANDU; SAGNELLA; MACGREGOR, 2001). A redução da ingestão de sal de 2$3 \mathrm{~g} /$ dia está associada à redução da pressão sistólica de 1,1 mm $\mathrm{Hg}$ em indivíduos normotensos e de 4,1 mm Hg em hipertensos (HE; LI; MACGREGOR; 2013). Além disso, associa-se à redução de $29 \%$ de acidentes cardiovasculares (em normotensos) e à diminuição de $31 \%$ de mortalidade cardiovascular (em hipertensos) (TAYLOR; ASHTON; MOXHAM; HOOPER; EBRAHIM, 2011b), estimando-se com esta medida, em indivíduos com mais de 35 anos, uma redução anual de acidentes cerebrovasculares de 5$16 \%$ e de mortalidade total de $3-13 \%$ (BIBBINSDOMINGO; CHERTOW; COXSON; MORAN; LIGHTWOOD; PLETCHER, 2010). Estimativas americanas apontam para uma redução dos acidentes cerebrovasculares de $24 \%$ e eventos coronários de $18 \%$ com uma redução de ingestão de sal de 6 g/dia (HE; JENNER; MACGREGOR, 2010). Na Finlândia, no período entre 1977 e 2002, associou-se a redução de 6 g/dia de sal a uma redução dos acidentes cerebrovasculares e da mortalidade cardiovascular de $60 \%$ (KARPPANEN; MERVAALA, 2006).

Os especialistas têm proposto uma mudança de políticas para melhorar o controlo da HTA. Entre outras medidas, a redução do consumo de sal constitui uma possível estratégia que pode ser promovida desde a administração de forma economicamente viável. Esta estratégia iniciou-se, basicamente, em 2004, com a Adoção da Estratégia Global da OMS sobre Dieta, Atividade Física e Saúde. Em outubro de 2006, a OMS apelou aos países para reduzirem a ingestão de sal para valores menores ou iguais a $5 \mathrm{~g} / \mathrm{dia}$ (ou $2000 \mathrm{mg} \mathrm{de} \mathrm{Na}$ ), diminuindo o consumo de sal em 30\% até 2025 (WHO, 2007). Em 2008 foi criado o quadro concetual (framework) da UE para as iniciativas nacionais de redução do consumo de sal.

Neste contexto, a Direção-Geral da Saúde (DGS) de Portugal divulgou, em 2013, o "Relatório Nacional para a Redução do Consumo de Sal na Alimentação". No 
documento, são apontadas estratégias gerais e a estratégia nacional de abordagem ao consumo excessivo de sal, sendo um dos objetivos estratégicos a sensibilização e a capacitação dos consumidores para um consumo reduzido de sal. Destaca-se o "Programa Nacional para a Promoção da Alimentação Saudável” (PORTUGAL-DIREÇÃOGERAL SAÚDE, 2015a) e o livro "Alimentação Inteligente-coma melhor, poupe mais" (PORTUGALDIREÇÃO-GERAL SAÚDE, 2015b). Outro objetivo operacional do Relatório consiste em "Promover a melhor forma de disponibilizar rotulagem capaz de destacar o conteúdo de sal dos alimentos e identificar produtos com pouco sal". A este nível, algumas empresas têm vindo a apresentar de forma facultativa e simplificada esta informação utilizando cores. Mais recentemente, uma diretiva comunitária, já transposta para Portugal, obriga a que todos os fabricantes coloquem esta informação

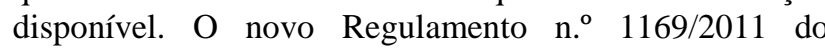
Parlamento Europeu e do Conselho, de 25 de outubro de 2011, torna a rotulagem nutricional obrigatória, e instrui os fabricantes de alimentos para fornecer informações sobre o valor da energia e 6 nutrientes: gordura, AGS, glícidos, proteína e sal - nesta ordem, e expressa por 100 g ou $100 \mathrm{~mL}$ de produto. Todos os produtos alimentares pré-embalados vendidos na UE ficaram obrigados, desde dezembro de 2014, a apresentar informações nutricionais de acordo com as novas regras.

A DGS divulgou, ainda, em 2013, um documento informativo "Utilização de Ervas Aromáticas \& Similiares na Alimentação", que reforça a utilização de ervas aromáticas como substituto do sal, exemplo seguido por muitos outros organismos no mundo (TAPSELL; HEMPHILL; COBIAC; PATCH; SULLIVAN; FENECH; ET AL., 2006), não sem que em alguns locais se alerte para o risco da intoxicação por incapacidade de reconhecimento das plantas, quando colhidas diretamente no campo. O documento carateriza 19 ervas, as suas propriedades terapêuticas e os seus usos culinários.

Em Portugal, num estudo realizado através do único método validado para determinação do consumo diário de sal, que se baseia no doseamento de Na na urina de 24 h com controlo da qualidade da colheita através da creatinúria, Polónia (2012) encontrou um consumo médio de sal de 12 g/dia (a Espanha tem 10,1 g/dia, o Reino Unido 8,9 g/dia e a Dinamarca 7,8 g/dia). E quando se procede à análise dos padrões alimentares de um subgrupo dessa população de doentes que foi sujeita ao estudo, percebe-se que alguns alimentos (pão, enchidos, queijos, produtos de charcutaria) são responsáveis, maioritariamente, por este elevado consumo de sal, para além da adição de sal na confeção ou na mesa.

O estudo de Magalhães (2008), e outros da mesma natureza, contribuíram para o estabelecimento das novas normas com vista à redução do teor de sal no pão bem como informação na rotulagem de alimentos embalados destinados ao consumo humano (Lei $n$. ${ }^{\circ}$ 75/2009, de 12 de agosto). O teor máximo permitido para o conteúdo de sal no pão, após confecionado, é atualmente de $1,4 \mathrm{~g}$ por $100 \mathrm{~g}$ de pão (ou o correspondente $0,55 \mathrm{~g}$ de Na por $100 \mathrm{~g}$ de pão).

A ingestão de uma dieta rica em frutas e verduras também apresenta um efeito hipotensor, que é independente do causado pela redução do consumo de sal.
Uma vez que a maior parte do $\mathrm{Na}$ da dieta provém do sal que os alimentos já contêm, particularmente aqueles processados a nível industrial, é recomendado seguir uma dieta baseada em alimentos não processados e com baixo nível de $\mathrm{Na}$ e moderação ao adicionar sal para condimentar os alimentos preparados em casa, com níveis de ingestão de sal situados abaixo dos $5 \mathrm{~g}$ diários.

O potássio $(\mathrm{K})$ é um nutriente essencial para o organismo humano. Atua no balanço e na distribuição da água no organismo, na condução de estímulos nervosos e na contração muscular, na manutenção do equilíbrio ácido-base e nos processos de produção de energia, além de interferir nos batimentos cardíacos. A suplementação alimentar de $\mathrm{K}$ tem sido associada a importante efeito protetor cardiovascular. As necessidades diárias em $\mathrm{K}$ de um adulto são de cerca de 4,7 g. Recentemente tem sido associada uma redução de risco de acidente vascular cerebral (AVC) de $11 \%$ e uma redução de 5-3 mm Hg da pressão arterial (HANKEY, 2012) à ingestão suplementar de $1 \mathrm{~g}$ de $\mathrm{K} / \mathrm{dia}$.

Em 2004, a Food and Nutrition Board of the Institute of Medicine definiu os valores de referência de ingestão de $\mathrm{K}$, que oferecem redução de risco das patologias associadas ao consumo de sal (tabela 1).

$\mathrm{Na}$ tabela 2 apresentam-se alguns exemplos de alimentos que contêm $\mathrm{K}$ e os seus respetivos teores aproximados.

Recentemente foi disponibilizado em Portugal um sal de $\mathrm{K}$, consubstanciando uma alternativa à utilização do $\mathrm{NaCl}$. Devem, porém, acautelar-se as contraindicações, como seja o caso de insuficiência renal grave e as precauções em doentes medicados com diuréticos poupadores de $\mathrm{K}$ ou moduladores do eixo renina-angiotensina-aldosterona.

\section{Dieta e hipercolesterolemia}

Os níveis de triglicerídeos (TRIG) normais variam de acordo com idade e o sexo. Um nível elevado de TRIG combinado com níveis baixos de lipoproteínas de baixa densidade $(L D L)$ e um nível alto de lipoproteínas de alta densidade $(H D L)$ parece acelerar a aterosclerose, aumentando o risco de ataque cardíaco e de AVC.

O colesterol (e os seus derivados) e a vitamina D são os esteroides mais importantes. O colesterol é um componente da membrana celular em animais, atuando como precursor na biossíntese dos esteroides biologicamente ativos, como sejam as hormonas esteroides e os ácidos e sais biliares. Existe também em algas vermelhas, na batateira e em pequenas quantidades em muitas outras plantas. É insolúvel em água e, concomitantemente, insolúvel no sangue, que o transporta no plasma sanguíneo, em todos os animais. O transporte de lípidos, principalmente TRIG e colesterol, entre órgãos e tecidos, é realizado por uma família de partículas designadas de lipoproteínas.

O colesterol existe tanto na forma livre como esterificada. É abundante nos tecidos que mais sintetizam ou que têm membranas densamente agrupadas em maior número (e.g., sangue, fígado, medula espinal, cérebro). $\mathrm{O}$ organismo humano tem cerca de $2 \mathrm{~g}$ de colesterol por cada quilograma de peso. Sem colesterol não existiria vida. Porém, o excesso de colesterol (hipercolesterolemia) 
constitui um dos principais fatores de risco, amplamente conhecido, para o desenvolvimento de DCV (LIPID RESEARCH CLINICS PROGRAM, 1984).

As lipoproteínas são associações entre proteínas e lípidos. A lipoproteína menos densa é designada de quilomícron, sendo transportadora de triacilglicerol. O triacilglicerol endógeno pode ser transportado, também, por lipoproteínas de muito baixa densidade $(V L D L-c)$. As lipoproteínas de densidade intermédia $(I D L-c)$ formam-se na transformação do $V L D L-c$ em $L D L-c$. As $L D L-c$ são as principais transportadoras de colesterol, constituindo $3 / 4$ do total do colesterol plasmático, embora perfazendo apenas $7 \%$ do colesterol total (CT). A função primordial é a de fornecer colesterol para células com recetores de $L D L-c$, como aqueles existentes nas glândulas suprarrenais, nos músculos esqueléticos, nos linfócitos, nas gónadas e nos rins.

Níveis elevados de $L D L$ aumentam o risco de DCV, em particular enfarte agudo do miocárdio, por formação de ateromas nas paredes das artérias. Níveis elevados de colesterol $I D L, V L D L$ e $L D L$ são considerados aterogénicos. Pelo contrário, níveis elevados de $H D L$ estão relacionados com menor progressão ou mesmo regressão de ateromas, retirando colesterol da circulação, estando associados à diminuição do risco de DCV.

Tabela 1 - Consumo adequado de K (LINUS PAULUS INSTITUTE, 2013).

\begin{tabular}{lcc}
\hline Estádio de vida & Idade & mg/dia \\
\hline Bebés & $0-6$ meses & 400 \\
& $7-12$ meses & 700 \\
Crianças & $1-3$ anos & 3000 \\
& $4-8$ anos & 3800 \\
Adolescentes & $9-13$ anos & 4500 \\
Adultos & $14-18$ anos & 4700 \\
Grávidas & 19 ou + anos & 4700 \\
Amamentação & $14-50$ anos & 4700 \\
\hline
\end{tabular}

Tabela 2 - Exemplos de alimentos que contêm K, e os seus respetivos teores aproximados (WHO, 2012).

\begin{tabular}{lcc}
\hline Grupo de alimentos & $\begin{array}{c}\text { Conteúdo em K (aprox.) } \\
\text { mg/100 g peso fresco }\end{array}$ & Exemplos \\
\hline Feijões e ervilhas & 1300 & Feijão-frade, ervilhas pombo, feijão, inhame. \\
Nozes & 600 & Avelãs, nozes, castanha de caju, castanha do Brasil \\
Vegetais verdes & 550 & Espinafre, repolho, salsa \\
Outros produtos hortícolas & 300 & Tomates, pepinos, abóboras \\
Fruta & 300 & Bananas, papaias, tâmaras \\
Vegetais de raiz & 200 & Cenouras, cebolas, beterraba \\
\hline Uma lista mais completa pode ser encontrada nas Dietary Guidelines for Americans 2005 do United States Department of Agriculture (USDHHS \& \\
USDAD, 2008).
\end{tabular}

Pode suceder que o nível do CT se encontre nos limites normais e que os níveis de $H D L-c$ sejam igualmente baixos, o que, sob estas condições, conduziria a taxas altas de crescimento de ateromas. Por vezes usa-se a relação $H D L-C T$ para representar o nível de colesterol de uma pessoa. A relação obtém-se dividindo o nível de $H D L-c$ pelo CT. Por exemplo, se uma pessoa apresenta $210 \mathrm{mg} / \mathrm{dL}$ de CT e um nível de $H D L$ de $45 \mathrm{mg} / \mathrm{dL}$, a relação proporcional encontra-se 4,7:1. O objetivo é manter a relação abaixo de 5:1, numa razão ótima de 3,5:1. Os efeitos desta relação podem complicar-se mais se considerarmos a concentração relativa de dimetilarginina assimétrica $(A D M A)$ no endotélio. Níveis elevados de $A D M A$, associados a níveis altos de $L D L-c$, proporcionam um fator de risco aumentado para as DCV.

Cerca de $85 \%$ do colesterol que temos no nosso corpo é produzido pelo próprio organismo, principalmente no fígado $(1 / 3$ da produção) - colesterol endógeno. A restante parte é adicionada pela dieta - colesterol exógeno. Alguns estudos desenvolvidos em tribos africanas têm mostrado que a ingestão de grandes quantidades de gordura animal não aumenta necessariamente os níveis de CT no sangue, mantendo-os baixos. A evidência científica tem revelado que não se pode associar a subida do nível de colesterol no sangue com o aumento da ingestão de quantidades adicionais de alimentos ricos em colesterol, em adultos (RAVNSKOV, 2008) e em crianças (WEIDMAN; ELVEBACK; NELSON; HODGSON; ELLEFSON, 1978). Um estudo recente mostrou, por exemplo, que o colesterol da dieta de ovos aumenta o nível de $H D L-c$ em homens com excesso de peso que consomem uma dieta restrita em glícidos (MATUNGI, RATLIFF; PUGLISI; TORRES-GONZALEZ; VAISHNAV; LEITE; ET AL., 2008).

Os alimentos que têm gorduras animais possuem colesterol, enquanto os alimentos que não têm gorduras estão isentos de colesterol ou apresentam quantidades inexpressivas. Alguns dos alimentos que têm maior nível de colesterol são os ovos, a carnes de vaca e as carnes de aves (tabela 3 ).

Mesmo seguindo uma dieta vegetariana ou pobre em colesterol, os níveis de colesterol plasmáticos podem ser elevados, resultando de distúrbios do metabolismo. Assim, pequenas elevações dos níveis de colesterol podem ser corrigidas com a mudança de alguns dos hábitos alimentares, embora hipercolesterolemias severas exijam a associação com tratamento farmacológico, por exemplo, com estatinas. As estatinas monitorizam, primeiramente, o colesterol produzido pelo fígado, bloqueando a enzima responsável pela sua produção (hidroxi-metil-glutaril-CoA redutase).

Sobre o colesterol exógeno podemos ter alguma intervenção, função do estilo de vida adotado, particularmente associado à dieta, ao exercício e ao peso corporal. A ingestão de AGS (gordura procedente de produtos de origem animal, como sejam as carnes vermelhas e os lácteos) induze um aumento do CT e de $L D L$ - $c$; enquanto os AGP (azeite de sementes) e AGM (azeite de oliveira) produzem um incremento dos níveis de $H D L-c$ e uma diminuição dos níveis de TRIG, CT e $L D L$ - 
c. Além disso, as dietas ricas em AGM dão lugar a partículas de $L D L-c$ menos suscetíveis à oxidação e com menos capacidade aterogénica (ROYO-BORDONADA, 2004).

Os AGT cada vez estão mais presentes na nossa dieta, formando uma parte da extensa gama de produtos processados (restaurantes de comida rápida, molhos, cremes, bolachas, produtos de pastelaria, alimentos précozinhados, gelados, entre outros). Os AGT produzem uma descida do $H D L-c$ e um aumento do $L D L-c$, a lipoproteína-a e a razão CT:HDL-c. Além disso, a sua ingestão em quantidades elevadas pode provocar um incremento dos níveis plasmáticos de TRIG, facilitar a trombogénese e promover a resistência à insulina.

Os ácidos gordos (AG) ómega-3 são ácidos carboxílicos polinsaturados e não podem ser produzidos pelo corpo humano. A principal fonte alimentar é o pescado azul de águas profundas (e.g., albacora, atum, bacalhau, cação, cavala, salmão marinho, sardinha, peixe espada, entre outros). Os AG ómega-3 de cadeia curta apresentam poucos benefícios para a saúde, e encontramse em óleos extraídos de soja, de girassol e de milho, e em alguns vegetais, como brócolos, couve, espinafre e rúcula. A linhaça constitui a melhor fonte de ómega-3.

Os AG ómega-3 de cadeia longa estão associados ao aumento moderado do tempo da hemorragia e diminuem a capacidade trombogénica do sangue, possuem um possível efeito antiarrítmico e reduzem os TRIG plasmáticos. Em doses relativamente altas reduzem a pressão arterial.

Por último, os estudos mais recentes indicam que a ingestão em quantidades elevadas de açúcares adicionados diminui os níveis plasmáticos de $H D L-c$ e aumenta o de TRIg e de $L D L-c$.

\section{Dieta e doenças cardiovasculares}

Mais do que a relação entre a ingestão de determinados nutrientes e alimentos com alguns fatores de risco cardiovascular, como a HTA e a hipercolesterolemia, interessa conhecer a relação direta existente entre a dieta e a DCV. Um estudo recente revisou a evidência científica disponível, procedente de estudos de coortes e ensaios clínicos, sobre a relação causal entre diferentes componentes da dieta (nutrientes, alimentos e padrões alimentares) e a doença coronária (MENTE; DE KONING; SHANNON; ANAND, 2009). De acordo com esta revisão sistemática, existe evidência sólida para se afirmar que o risco de doença coronária diminui com a ingestão de AGM, verduras, frutos secos e um padrão de dieta mediterrânica; enquanto aumenta com a ingestão de AGT, um padrão de dieta ocidental e dietas com carga glicémica alta. A evidência é moderada para um efeito protetor da ingestão de AG ómega-3 com origem no pescado, dos cereais industriais, do ácido fólico, das frutas, das vitaminas $\mathrm{C}$, E e $\beta$-caroteno procedente da dieta, fibra e álcool em quantidades moderadas. Por último, a evidência é débil sobre o possível efeito da ingestão de vitaminas $\mathrm{C}$ e $\mathrm{E}$ sob a forma de suplementos, AGS, AGP, carne, leite e ovos, pelo que convém ser prudente no momento de se fazerem recomendações sobre estes componentes da dieta, já que não se justifica manter algumas das sugestões que se fizeram no passado a este respeito, baseando-se na sua potencial relação com o risco cardiovascular.

Tabela 3 - Fontes alimentares de colesterol listadas em ordem decrescente. Média = $270 \mathrm{mg}$.

\begin{tabular}{lccc}
\hline Grupo de alimentos & Ranking & Percentagem total & Percentagem cumulativa \\
\hline Ovos & 1 & 29,3 & 29,3 \\
Carne de vaca & 2 & 16,1 & 45,4 \\
Carnes de aves domésticas & 3 & 12,2 & 57,6 \\
Queijo & 4 & 5,8 & 63,4 \\
Leite & 5 & 5,0 & 68,4 \\
Peixe/Marisco & 6 & 3,7 & 72,1 \\
Bolos/biscoitos & 7 & 3,3 & 75,4 \\
Porco (não transformado) & 8 & 2,8 & 78,2 \\
Gelado/Iogurte & 9 & 2,5 & 80,7 \\
Salsicha & 10 & 2,0 & 82,7 \\
\hline
\end{tabular}

Fonte: USDHHS \& USDAD (2008).

\section{Recomendações a nível individual}

Em função do conjunto da evidência científica existente e disponível, a melhor forma de prevenir as doenças crónicas mais prevalentes do nosso tempo, como a obesidade, as DCV e o cancro, que causam aproximadamente $2 / 3$ do total das mortes, poderia resumir-se em três recomendações:

a) manter o peso dentro nível considerado saudável, evitando tanto o excesso como o baixo peso;

b) levar uma vida fisicamente ativa, realizando atividade física de forma regular e restringindo as atividades sedentárias; e

c) adotar um padrão alimentar saudável, como a dieta mediterrânica ou outra de caraterísticas similares.

\section{Recomendações a nível coletivo}

Os hábitos alimentares têm a ver com decisões pessoais que afetam o âmbito da responsabilidade individual, num cenário onde cada pessoa pode escolher livremente a forma de se alimentar. Porém, para que uma pessoa possa escolher livremente exigem-se, no mínimo, duas condições: em primeiro lugar, deveria dispor, única e exclusivamente, de informação fiável, obtida a partir de evidências científicas razoavelmente sólidas, ao seu alcance sobre os efeitos na saúde dos produtos alimentares que o mercado dispõe, num formato e meio adaptados às suas condições pessoais, de forma que esta seja compreensível e facilmente acessível. Em segundo lugar, as opções mais saudáveis deveriam ser facilmente acessíveis para todas as pessoas, independentemente do seu estado de saúde, da sua posição 
socioeconómica, do seu nível cultural, e do local onde vivem. Contudo, por vezes, a informação não se encontra facilmente acessível ou não está disponível num formato compreensível para todo o cidadão. Por exemplo, a rotulagem nutricional não proporciona informação sobre os AGT e não é facilmente compreendida por alguns segmentos da população. Em outras ocasiões, a informação disponível não é fiável, ao não existirem provas científicas sólidas daquilo que se afirma (e.g., determinados produtos fazem alegações de saúde que não foram comprovadas pela autoridade correspondente). Por último, não é raro o uso de técnicas de marketing fraudulentas, que não respeitam os códigos éticos sobre a publicidade do uso. Além disso, em determinadas ocasiões o meio dificulta mais do que facilita as opções saudáveis (e.g., a presença de máquinas de distribuição automática de alimentos e bebidas em estabelecimentos de ensino; ausência de fontes de água; menus escolares não elaborados com base em critérios nutricionais). Consequentemente, além da responsabilidade individual, existe uma responsabilidade coletiva de construir um meio sociopolítico, físico, económico e cultura que facilite as opções saudáveis, para que cada pessoa esteja em condições de escolher livremente a forma de se alimentar, em função dos seus próprios interesses e preferências. Entre as medidas que se poderiam realizar para alcançar este objetivo, figuram garantir a disponibilidade de informação fiável e acessível e construir um meio que facilite as opções saudáveis.

\section{Pirâmide alimentar e dieta mediterrânica}

A dieta mediterrânica, que tem sido tradicionalmente a base do padrão alimentar em Portugal, merece uma menção especial. Em 2010 foi declarada Património Cultural Imaterial da Humanidade pela UNESCO. Esta decisão assenta na essencialidade da dieta mediterrânica como um conjunto de práticas tradicionais, conhecimentos e habilidades transmitidas de geração em geração e que proporciona um sentimento de pertença e de continuidade para as comunidades envolvidas, e o facto de se poder vir a dar uma maior visibilidade à diversidade do património cultural imaterial e promove o diálogo intercultural a nível regional e internacional. Em 2013 a classificação obtida foi alargada a outros Estados e comunidades: Portugal, Croácia, Chipre, Espanha, Marrocos, Itália e Grécia.

À dieta mediterrânica é indexada, com evidência científica, capacidade para prevenir as principais doenças crónicas, como as DCV e o cancro, aumentando assim a esperança e a qualidade de vida das populações onde está presente. Ainda assim, estudos recentes revelam que os países da bacia do mediterrâneo se têm vindo a afastar deste padrão alimentar, enquanto alguns países do Norte da Europa estão começando a adotar este tipo de dieta. As estratégicas imagéticas em educação para a saúde procuram informar, ajudar e motivar os consumidores a escolherem facilmente os alimentos no momento de decisão. Estes esforços, porém, não revelam como as pessoas compõem um prato de dieta saudável. Amiúde, usam-se critérios baseados em ciência fora-do-tempo e influências de pessoas com interesses comerciais nas mensagens dos ícones transmitidos.

Em 2010, a Fundación Dieta Mediterránea, com a colaboração de numerosas entidades internacionais e um amplo grupo de especialistas pertencentes a diversas disciplinas, chegaram a um consenso de um novo esquema que enriquece a pirâmide tradicional da dieta mediterrânica, com a incorporação de novos elementos qualitativos. Seguindo o princípio da Healthy Eating Pyramid, a Pirâmide da Dieta Mediterrânica (PDM) (figura 1) coloca na base os alimentos que devem ser o sustento da dieta, deixando nas camadas superiores, graficamente mais estreitas, aqueles que se devem consumir com moderação. Além disso, introduz indicações de ordem cultural e social, intimamente ligadas ao estilo de vida mediterrânico, a partir de um conceito de dieta entendida no seu sentido mais amplo. Reflete, ainda, a composição e o número de porções das principais comidas. A PDM estabelece, também, critérios alimentares de cumprimento diário, semanal e ocasional, para uma dieta saudável e equilibrada.

Façamos, de seguida, um périplo pela PDM. As refeições principais não devem prescindir de três elementos básicos: a) cereais - uma ou duas porções por refeição, em forma de pão, massas, arroz, cereais, ou outros. Devem ser, preferencialmente, integrais para que alguns nutrientes (e.g., $\mathrm{Mg}, \mathrm{P}$, entre outros) e fibras não se percam no processado; b) verduras - devem fazer parte do almoço e do jantar, em cerca de duas doses em cada refeição. Uma das porções deve ser crua. Recomenda-se a utilização de vários tipos de verduras (cores e texturas), porque fornecem uma diversidade de antioxidantes e substâncias protetoras; e c) frutas - uma ou duas porções por refeição - que deve ser a sobremesa habitual. A água deve estar sempre presente como meio preferencial de hidratação, ingerindo-se 1,5-2 L/dia, necessidades que variam consoante a idade das pessoas, o nível de atividade física que desenvolvam, a situação pessoal e as condições climáticas. A ingestão de água pode ser complementada com infusões de ervas com açúcar moderado ou caldos com pouca gordura e reduzida quantidade de sal.

Figura 1 - Pirâmide da Dieta Mediterrânea portuguesa (FUNDACIÓN DIETA MEDITERRÁNEA, 2015).

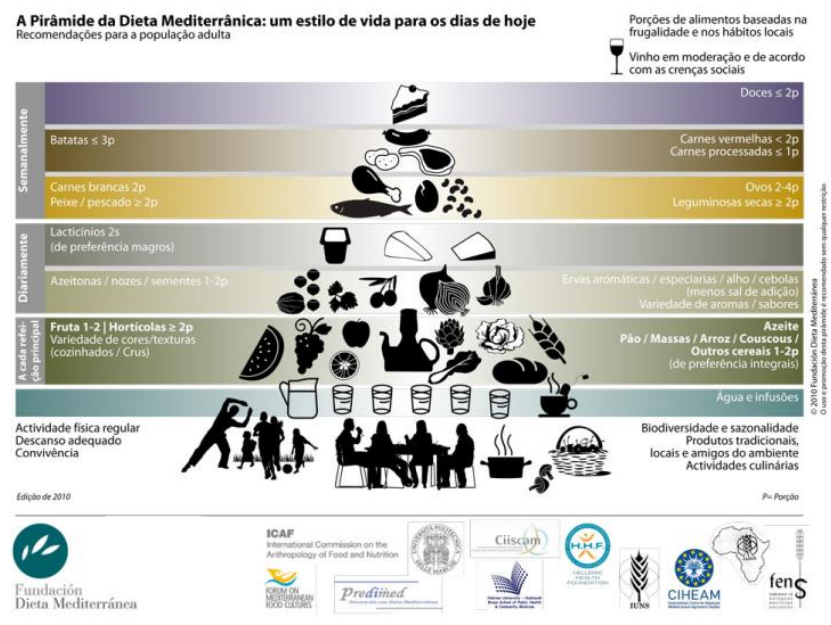

A adoção do padrão alimentar da dieta mediterrânea pressupõe a consideração de alguns elementos-chave: a) Moderação - A dimensão das porções deve basear-se na temperança, uma vez que o estilo de vida sedentário que carateriza as sociedades urbanas requer baixas necessidades energética; b) Confeção - Dedicar tempo à preparação dos alimentos para que o ato de cozinhar traduza uma atividade relaxada, prazenteira, e que o confecionador se divirta pessoalmente com a família e com amigos; c) Socialização A convivência ao redor da comida potencia o valor social e 
cultura. Sentar-se em redor da mesa e partilhar a refeição com a família e de forma amistosa proporciona um sentido de comunidade; d) Sazonalidade - Os alimentos próprios do tempo, frescos e minimamente processados têm mais nutrientes e substâncias protetoras que os alimentos fora do tempo. Deve dar-se prioridade aos produtos tradicionais, locais, respeitando o meio ambiente e a biodiversidade, contribuindo para a sua preservação e das paisagens mediterrânicas; e) Atividade - Está recomendada prática de atividade física moderada (mínimo de $30 \mathrm{~min}$ no decurso do dia), trazendo benefícios para a saúde, como gasto regular de energia e a manutenção do peso corporal saudável. Atividades ao ar livre e em companhia potenciam o atrativo do exercício físico e reforçam os vínculos familiares e com a comunidade; f) Descanso - É fundamental descansar adequadamente como forma de um estilo de vida saudável e equilibrado, com um período de sono entre as 7 e as 9 horas diárias, de forma seguida.

\section{CONSIDERAÇÕES FINAIS}

Ao londo desta revisão da bibliografia procurámos evidências científicas sobre a relação entre a dieta mediterrânica e a HTA, a hipercolesterolemia e as DCV. A dieta mediterrânica carateriza-se pela abundância de produtos de origem vegetal (frutas, verduras, cereais, legumes, frutos secos, entre outros), frescos ou minimamente processados, a escassez de produtos ricos em açúcares refinados e carnes vermelhas, a presença de azeite de oliveira como principal fonte de gordura, a ingestão de queijo, iogurte, frango e pescado em quantidade moderadas e o consumo de vinho às refeições (WILLETT; SACKS; TRICHOPOULOU; DESCHER; FERRO-LUZZI; HELSING; TRICHOPOULOS, 1995). Os mecanismos biológicos dos efeitos cardiosaudáveis e quimiopreventivos da dieta mediterrânica relacionam-se com o seu equilíbrio em AG essenciais das séries ómega-3 e ómega-6 e o seu alto conteúdo em AGM, fibra, antioxidantes, polifenois e flavonoides procedentes da fruta, a verdura, os legumes, o azeite de oliveira e o vinho. A dieta mediterrânica associa-se a $9 \%$ de redução na mortalidade total, principalmente por DCV, mas também por cancro e AVC (FOROUGHI; AKHAVANZANJANI; MAGHSOUDI; GHIASVAND; KHORVASH; ASKARI, 2013) e com $13 \%$ de redução na incidência das doenças de Parkinson e Alzheimer (SOFI; CESARI; ABBATE; GENSINI; CASINI, 2008).

A dieta mediterrânica constitui, portanto, um padrão alimentar ideal para prevenir a aparição das principais doenças crónicas do nosso tempo, que contribui para prolongar os anos de vida em bom estado de saúde.

\section{REFERÊNCIAS BIBLIOGRÁFICAS}

ALDERMAN, M. Salt, Blood Pressure, and Human Health. Hypertension, n. 36, p. 890-893, 2000. Disponível em: <http://hyper.ahajournals.org/content/36/5/890.full.pdf+html>

BIBBINS-DOMINGO, K.; CHERTOW, G.; COXSON, P.; MORAN, A.; LIGHTWOOD, J.; PLETCHER, M.; et al. Projected Effect of Dietary Salt Reductions on Future Cardiovascular Disease. New england journal of medicine, $n$.
362, p. 590-599, 2010. Disponível em: < http://www.nejm.org/doi/full/10.1056/NEJMoa0907355>.

PORTUGAL - DIREÇÃO-GERAL DA SAÚDE. Plataforma obsesidade, 2015a. Disponível em: <http://www.plataformacontraaobesidade.dgs.pt/Presentation Layer/conteudo.aspx?menuid $=115 \&$ exmenuid $=114>$.

O livro "Alimentação inteligente" - Coma melhor, poupe mais" foi o vencedor do Prémio Nutrition Awards 2013, 2015b. Disponível em: <http://www.plataformacontraaobesidade.dgs.pt/Presentation Layer/conteudo.aspx ?menuid $=115 \&$ exmenuid $=114>$.

DURÃO, C.; OLIVEIRA, J.; ALMEIDA, M. Portugal e o padrão alimentar mediterrâneo. Revista de alimentação humana, v. 14, n. 3, p. 115-128, 2008. Disponível em: $<$ http://www.spcna.pt/download.php?path=pdfs\&filename $=\mathrm{S}$ PCNA_20100123134305_RAH_2008_n_3_3.pdf >.

EFSA - EUROPEAN FOOD SAFETY AUTHORITY. Scientific opinion on dietary reference values for fats, including saturated fatty acids, polyunsaturated fatty acids, monounsaturated fatty acids, trans fatty acids, and cholesterol. EFSA Panel on Dietetic Products, Nutrition, and Allergies (NDA). EFSA journal, v. 8, n. 3, p. 1461, 2010. Disponível em: <http://www.efsa.europa.eu/en/efsajournal/doc/1461.pdf>.

FOROUGHI, M.; AKHAVANZANJANI, M.; MAGHSOUDI, Z.; GHIASVAND, R.; KHORVASH, F.; ASKARI, G. Stroke and nutrition: a review of studies. International journal of preventive medicine, n. 4(Suppl 2), p. S165-S179, 2013. Disponível em: <http://www.ncbi.nlm.nih.gov/pmc/articles/PMC3678213/?re port=printable $>$.

FREEDMAN, D.; PETITTI, D. Salt and blood pressure. Conventional wisdom reconsidered. Evaluation review, v. 25, n. 3, p. 267-287, 2001. Disponível em: <http://www.stat.berkeley.edu/ census/573.pdf>.

FUNDACIÓN DIETA MEDITERRÁNEA, 2015. Disponível em:

<http://dietamediterranea.com/dietamed/piramide_PORTUG UES.pdf>.

HANKEY, G. Nutrition and the risk of stroke. The lancet neurology, v. 11, n. 1, p. 66-81, 2012. Disponível em: <http://www.natap.org/2011/newsUpdates/PIIS14744422117 02654.pdf $>$.

HE, F.; JENNER, K.; MACGREGOR, G. WASH - world action on salt and health. Kidney international, v. 78, n. 8, p. 745-753, 2010. Disponível em: <http://www.ncbi.nlm.nih.gov/pubmed/20720531>.

HE, F.; LI, J.; MACGREGOR, G. Effect of longer term modest salt reduction on blood pressure: Cochrane systematic review and meta-analysis of randomised trials. British medical journal, n. 346, fl325, 2013. Disponível em: <http://www.ncbi.nlm.nih.gov/pubmed/23558162>. 
HE, F.; MARKANDU, N.; SAGNELLA, G.; MACGREGOR, G. Effect of Salt Intake on Renal Excretion of Water in Humans. Hypertension, n. 38, p. 317-320, 2001. Disponível em: <http://hyper.ahajournals.org/content/38/3/317.full.pdf+html>

KARPPANEN, H.; MERVAALA, E. Sodium intake and hypertension. Progress in cardiovascular diseases, v. 49, n. 2 , p. 59-75, 2006. Disponível em: <http://www.drtsoukalas.com/uploads/file/SODIUM\%20INT AKE\%20HYPERTENSION\%20FINLAND.pdf $>$.

LIMA, M.; ROSENTHAL, A.; DELIZA, R. Semáforo nutricional (traffic light labelling): Uma alternativa para melhores escolhas alimentares. Alimentação humana, v. 20, n. 2 e 3, p. 39-46, 2014.

LINUS PAULUS INSTITUTE. Micronutrient information Center, 2013. Disponível em: <http://lpi.oregonstate.edu/infocenter/minerals/potassium/>.

LIPID RESEARCH CLINICS PROGRAM. The Lipid Research Clinics Coronary Primary Prevention Trial Results. 11. The relationship of reduction in incidence of coronary heart dis- ease to cholesterol lowering. Journal of american medicine association, n. 251, p. 365-374, 1984. Disponível em: <http://www.jlr.org/content/31/8/1413.full.pdf>.

LONGO-SILVA, G.; TOLONI, M.; TADDEI, J. Traffic light labelling: traduzindo a rotulagem de alimentos. Revista de nutrição, v. 23, n. 6, p. 1031-1040, 2010. Disponível em: <http://www.scielo.br/pdf/rn/v23n6/09.pdf >.

MAGALHÃES M. Avaliação dos padrões alimentares determinantes dos níveis diários da excreção urinária de sódio em doentes hipertensos. Porto: Faculdade de Medicina da Faculdade do Porto, 2008. Disponível em: <http://repositorioaberto.up.pt/bitstream/10216/22408/4/Dissertao.pdf $>$.

MATUNGI, G.; RATLIFF, J.; PUGLISI, M.; TORRESGONZALEZ, M.; VAISHNAV, U.; LEITE, J.; et al. Dietary Cholesterol from Eggs Increases Plasma HDL Cholesterol in Overweight Men Consuming a Carbohydrate-Restricted Diet. Journal of nutrition, v. 138, n. 2, p. 272-276, 2008. Disponível

em:<http://jn.nutrition.org/content/138/2/272.full.pdf+html>.

MENTE, A.; DE KONING, L.; SHANNON, H.; ANAND, S. A systematic review of the evidence supporting a causal link between dietary factors and coronary heart disease. Arch intern med., v. 169, n. 7, p. 659-669, 2009. Disponível em: <http://www.dcscience.net/mente-aim-2009.pdf>.

MONTEIRO, I.; ALMEIDA, M. D. V. Gordura alimentar e risco. De acidente vascular cerebral isquémico no norte de Portugal. Acta médica <portuguesa, n. 20, p. 307-317, 2007. Disponível em:

http://actamedicaportuguesa.com/revista/index.php/amp/articl e/viewFile/871/545>.

BRASIL - MINISTÉRIO DA SAÚDE. Guia alimentar para a população brasileira: promovendo a alimentação saudável. Brasília, DF: Ministério da Saúde, 2008. Disponível em: <http://bvsms.saude.gov.br/bvs/publicacoes/guia_alimentar_p opulacao_brasileira_2008.pdf >

POLÓNIA J. Consumo excessivo de sal. Riscos de uma toxicodependência e formas de a combater. Revista portuguesa de hipertensão e risco cardiovascular, n. 31, p. 1013, 2012. Disponível em: <http://www.sphta.org.pt/pdf/SPHTA_31_2012_0910.pdf>.

RAVNSKOV U. Should medical science ignore the past? British medicine journal, n. 337, a1681, 2008. Disponível em: <http://www.bmj.com/content/337/bmj.a1681.full?rss=1>.

ROYO-BORDONADA, M. Recomendaciones nuricionales y alimentarias para una dieta cardiosaludable. Revista española de nutrición comunitaria, v. 10, n. 3, p. 122-143, 2004. Disponível em: <http://www.nutricioncomunitaria.org/BDProtegidos/n-10-3005_I_1143674167011.pdf>.

STENDER, S.; DYERBERG, J. High hevels of industrially produced trans fat in popular fast foods. The new england journal of medicine, v. 354, n. 15, p. 1650-1652, 2006. Disponível <http://www.nejm.org/doi/pdf/10.1056/NEJMc052959>.

SOFI, F.; CESARI, F.; ABBATE, R.; GENSINI, G.; CASINI, A. Adherence to mediterranean diet and health status: metaanalysis. British medical journal, n. 337, p. a1344, 2008. Disponível em: <http://www.bmj.com/content/337/bmj.a1344.pdf\%2Bhtml>.

TAPSELL, L.; HEMPHILL, I.; COBIAC, L.; PATCH, C.; SULLIVAN, D.; FENECH, M.; et al. Health benefits of herbs and spices: the past, the present, the future. Medical journal of Australia, v. 185, n. 4, Suppl. S4-S24, 2006. Disponível em: <http://digital.library.adelaide.edu.au/dspace/bitstream/2440/ 22802/1/hdl_22802.pdf >.

TAYLOR, R.; ASHTON, K.; MOXHAM, T.; HOOPER, L.; EBRAHIM, S. Reduced Dietary Salt for the Prevention of Cardiovascular Disease: A Meta-Analysis of Randomized Controlled Trials (Cochrane Review). American journal of hypertension, v. 24, n. 8, p. 843-853, 2011a. Disponível em: <http://ruhlman.com/wpcontent/uploads/2011/07/ajh2011115a.pdf>.

Reduced Dietary Salt for the Prevention of Cardiovascular Disease. Cochrane database system review, v. 6, n. 7, CD009217, 2011b. Disponível em: <http://www.ncbi.nlm.nih.gov/pubmed/21735439>.

USDHHS \& USDAD - U.S. DEPARTMENT OF HEALTH AND HUMAN SERVICES, \& U.S. DEPARTMENT OF AGRICULTURE. Dietary guidelines for americans 2005. 6th ed. Washington, DC: Government Printing Office, 2008. Disponível

em: <http://www.health.gov/dietaryguidelines/dga2005/document /pdf/DGA2005.pdf>.

WARDENER, H.; MACGREGOR, G. Harmful effects of dietary salt in addition to hypertension. Journal of human hypertension, v. 16, n. 4, p. 213-223, 2002. Disponível em: 

f>.

WEIDMAN, W.; ELVEBACK, L.; NELSON, R.; HODGSON, P.; ELLEFSON, R. Nutrient intake and serum cholesterol level in normal children 6 to 16 years of age. Pediatrics, v. 61, n. 3, p. 354-359, 1978. Disponível em: <http://www.ncbi.nlm.nih.gov/pubmed/205827>.

WILLETT, W.; SACKS, F.; TRICHOPOULOU, A.; DESCHER, G.; FERRO-LUZZI, A.; HELSING, E.; TRICHOPOULOS, D. Mediterranean diet pyramid; a cultural model for healthy eating. American journal of clinical nutricion, n. 61(suppl), p. 1402S-1406S, 1995. Disponível em: <http://ajcn.nutrition.org/content/61/6/1402S.full.pdf>.

WHO - WORLD HEALTH ORGANIZATION. Reducing salt intake in populations: report of a WHO forum and technical meeting. Geneva: World Health Organization, 2007. Disponível em: <http://www.who.int/dietphysicalactivity/Salt_Report_VC_ap ril07.pdf $>$.

WHO - WORLD HEALTH ORGANIZATION. Set of recommendations on the marketing of foods and nonalcoholic beverages to children. Geneva: World Health Organization, 2010. Disponível em: <http://whqlibdoc.who.int/publications/2010/9789241500210 _eng.pdf>.

WHO - WORLD HEALTH ORGANIZATION. Guideline: Potassium intake for adults and children. Geneve: World Health Organization, 2012. Disponível em: <http://www.who.int/nutrition/publications/guidelines/potassi um_intake_printversion.pdf $>$. 